E.C. Arruda

https://orcid.org/0000-0001-7762-0302 R.C.M. Garcia

https://orcid.org/0000-0001-7333-013X S.T.O. Stedile

https://orcid.org/0000-0002-8218-379X

\title{
Bem-estar dos cães de abrigos municipais no estado do Paraná, Brasil, segundo o protocolo Shelter Quality
}

\author{
[The welfare of dogs from municipal shelters in the state of Paraná, Brazil, \\ under the Shelter Quality protocol] \\ E.C. Arruda ${ }^{1}$, R.C.M. Garcia ${ }^{2}$, S.T. Oliveira ${ }^{2}$ \\ ${ }^{1}$ Aluno de pós-graduação - Universidade Federal do Paraná - Curitiba, PR \\ ${ }^{2}$ Universidade Federal do Paraná - Curitiba, PR
}

\begin{abstract}
RESUMO
O bem-estar dos animais, além dos aspectos individuais, depende do entendimento e dedicação dos responsáveis pelo abrigo. O objetivo do presente trabalho foi avaliar o bem-estar dos cães mantidos em abrigos municipais do estado do Paraná, mediante a aplicação do protocolo Shelter Quality. Foram avaliados 16 abrigos, 439 cães e 165 recintos, na estação do outono de 2017. A maioria dos abrigos apresentou cães com condição corporal adequada e pelagem limpa, em recintos seguros, com metragem e suprimento de água adequados e conforto térmico. Além disso, a maioria dos cães eram mantidos em recintos coletivos e em ambientes abertos, com baixo nível de ruído e demonstravam mais emoções positivas, desejáveis na adoção. Como aspectos negativos, 58\% (96/165) dos recintos avaliados tinham camas inadequadas ou inexistentes e 30\% (50/165) dos recintos mantinham cães individualmente, sendo indicadores de baixo grau de bem-estar para os cães envolvidos. Além disso, nenhum abrigo disponibilizava ração para cães idosos e $81 \%$ (13/16) dos abrigos negligenciavam o diagnóstico e tratamento da dor. Apesar de a maioria dos abrigos apresentarem mais pontos positivos que negativos em sua avaliação, a gestão dos abrigos deve sempre visar à correção dos pontos críticos que comprometam o bem-estar dos animais.
\end{abstract}

Palavras-chave: canis, qualidade de vida, protocolo de avaliação

\begin{abstract}
Animal welfare, in addition to individual aspects, depends on the understanding and dedication of those responsible for the shelter. The objective of this study was to evaluate the welfare of dogs kept in municipal shelters in the state of Paraná, using the Shelter Quality protocol. A total of 16 shelters, 439 dogs and 165 enclosures were evaluated in the fall season of 2017. Most shelters presented dogs with adequate body condition and clean coat, in secure enclosures with adequate water supply and thermal comfort. In addition, most dogs were kept in collective enclosures and in open environments, with low noise levels and showing more positive, desirable emotions in adoption. As negative aspects, 58\% (96/165) of the precincts evaluated had inadequate or inexistent beds and 30\% (50/165) of the enclosures kept dogs individually, being indicators of a low degree of well-being for the dogs involved. In addition, no shelter provided elderly dog rations and $81 \%$ (13/16) of the shelters neglected the diagnosis and treatment of pain. Although most shelters present more positive than negative points in their evaluation, shelter management should always aim at correcting critical points that compromise animal welfare.
\end{abstract}

Keywords: pens, quality of life, evaluation protocol

\section{INTRODUÇÃO}

No Brasil, estimam-se 52,2 milhões de cães, representando 1,8 cão por domicílio, sendo a Região Sul com a maior proporção $(58,6 \%)$ de

Recebido em 18 de fevereiro de 2019

Aceito em 20 de março de 2019

E-mail: ecarruda@ufpr@gmail.com domicílios com pelo menos um cão (Acesso..., 2013). No entanto, no país não é estimado o número de cães soltos nas ruas, embora seja visivelmente elevado. Os cães soltos em vias públicas sem a supervisão humana, sejam eles semidomiciliados, abandonados ou perdidos, estão expostos a diversos riscos e maus-tratos. 
Esses cães ficam suscetíveis a doenças infecciosas e parasitárias, atropelamento, brigas, fome, sede, traumas não acidentais, como envenenamento e agressões físicas por pessoas, bem como ausência de atendimento veterinário (Guia..., 2007), comprometendo severamente seu grau de bem-estar (Hammerschmidt e Molento, 2012).

Os animais de rua no Brasil são tutelados pelo Estado (Calhau, 2007). A Constituição Federal de 1988, art. 225, foi a primeira no mundo a considerar a crueldade contra animais. Existe, ainda, a Lei de Crimes Ambientais n ${ }^{\circ}$ 9.605/98, art. 32 (Brasil, 1998), que prevê como crime os maus-tratos a animais, e, no Paraná, a Lei Estadual 14037/2003 estabelece medidas protetivas aos animais. Dessa forma, assim como sugere a Aliança Internacional para Controle de Animais de Companhia (Guia..., 2007), os abrigos devem ser uma das estratégias que compõem um programa de manejo humanitário, que tem o objetivo de recolher, reabilitar e reintroduzir os animais em risco em lares com guarda responsável.

Os abrigos públicos no Paraná, tais como Canis Municipais (CM) e Centros de Referência de Animais em Risco (Crar), têm uma proposta de prestar atendimento a esses animais em risco recolhidos. O Ministério da Saúde, por meio da Portaria $\mathrm{n}^{\mathrm{o}} 1.138 / 2014$, estabelece que as Unidades de Vigilância em Zoonoses (UVZ) realizem ações e serviços públicos de saúde voltados para a vigilância, a prevenção e o controle de zoonoses (Brasil, 2014). Dessa forma, as UVZs podem abrigar animais de rua suspeitos de doenças zoonóticas, cães e gatos agressivos com histórico de mordedura em pessoas e cavalos vítimas de maus-tratos ou de abandono em vias públicas. E, assim, independentemente da missão, todos esses estabelecimentos devem seguir os preceitos da medicina de abrigos.

No Paraná, todos os abrigos municipais, que mantêm cães, gatos e cavalos, funcionam como locais de adoção de animais (Arruda et al., 2019). Para que os cães mantidos em abrigos tenham um grau de bem-estar alto, suas liberdades nutricional, sanitária, ambiental, psicológica e comportamental devem ser atendidas (Guia..., 2016). Para tanto, fazem-se necessárias instalações adequadas para a espécie alojada, recursos disponíveis dentro dos recintos e boa gestão do estabelecimento.

A qualidade dos abrigos interfere no grau de bem-estar, no comportamento e na adoção desses animais. Dessa forma, é importante avaliar os animais e a qualidade dos abrigos, a fim de identificar pontos críticos que podem prejudicar os animais e que devem ser corrigidos. O Shelter Quality é uma ferramenta internacionalmente válida para esse fim, construído com base em quatro princípios (boa alimentação, boa acomodação, boa saúde, comportamento apropriado), que envolvem as cinco liberdades. Além disso, avalia, independentemente, as instalações, os recursos existentes e a gestão. Assim, o objetivo deste trabalho foi avaliar, por meio da aplicação do protocolo de avaliação Shelter Quality, o bem-estar dos cães mantidos em abrigos vinculados às prefeituras municipais do Paraná, e identificar os pontos críticos existentes nesses estabelecimentos, assim como sugerir medidas corretivas.

\section{MATERIAL E MÉTODOS}

O levantamento dos municípios que recolhem cães e que são vinculados ao poder público no estado do Paraná foi realizado junto ao Conselho Regional de Medicina Veterinária do Paraná. Foram verificados 17 municípios com abrigos municipais de cães; destes, 16 aceitaram participar da pesquisa, recebendo a visita de um dos autores do projeto, para aplicação do protocolo Shelter Quality. As visitas aos abrigos ocorreram nos meses de abril e maio de 2017, na estação do outono, nos períodos da manhã e da tarde, e foram previamente agendadas via ligação telefônica.

Todas as visitas e avaliações foram realizadas pelo mesmo pesquisador, com experiência na área, acompanhado de um auxiliar. Nas visitas, o pesquisador foi recebido por um médico veterinário ou funcionário, que responderam a um questionário de gestão do abrigo. Todos os participantes entrevistados assinaram um termo antes de se iniciar a avaliação dos abrigos, que esclarecia intenções, benefícios e riscos da pesquisa e se comprometia a manter sigilo sobre a identificação dos abrigos e dos municípios participantes. Em seguida, foi realizada a avaliação do abrigo, dos recintos e dos animais, por meio dos dados mensuráveis (Fig. 1). 


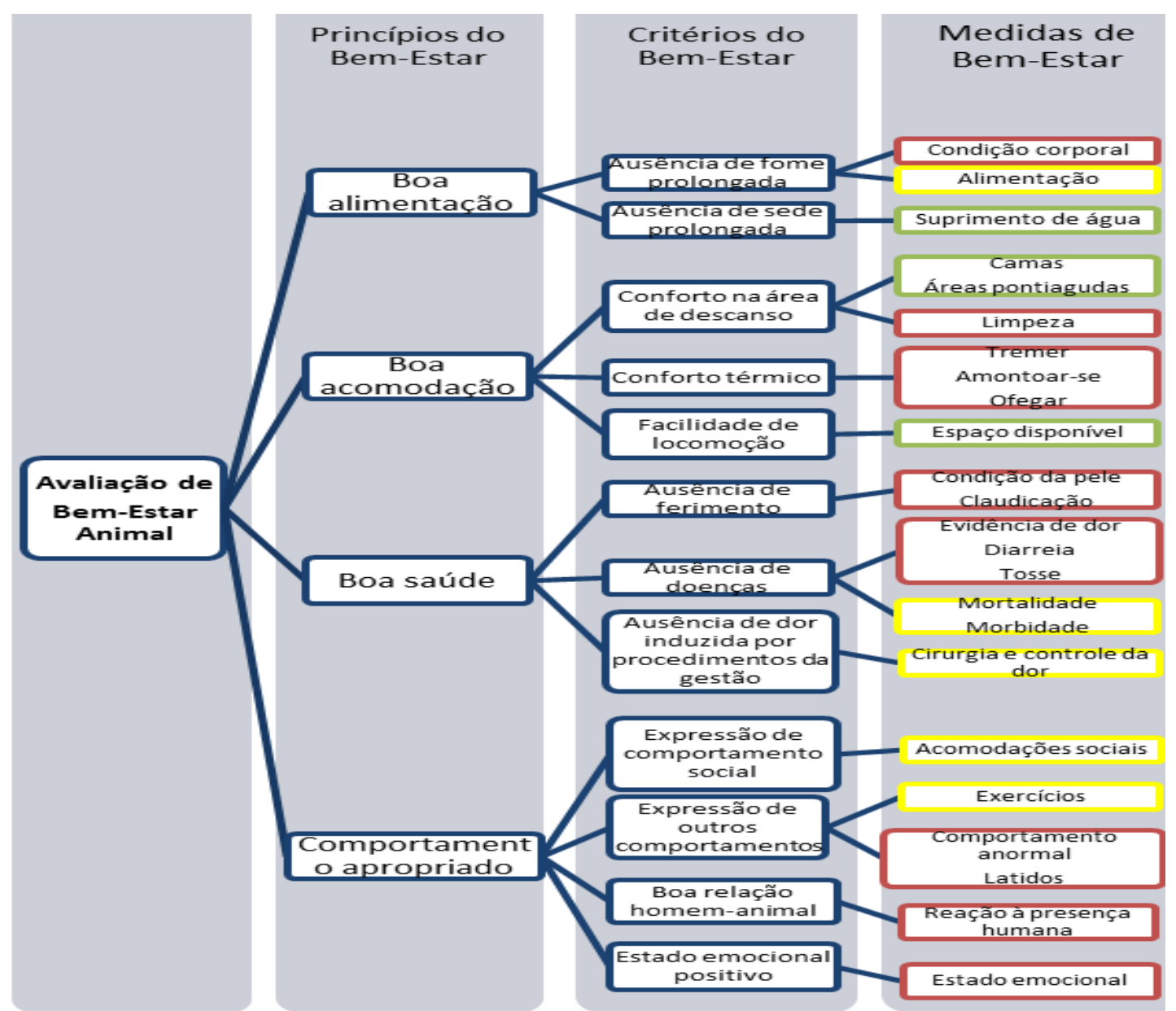

Figura 1. Medidas para avaliação do bem-estar animal, de acordo com o protocolo Shelter Quality. As mensurações de bem-estar foram realizadas em três níveis: amarelo (nível do abrigo), verde (nível dos recintos) e vermelho (nível individual dos cães).

A temperatura, a umidade e o nível de ruído foram mensurados com o Medidor Multifuncional THDLA 500. O protocolo Shelter Quality sugere aferir o nível de ruído pela escala análoga visual (EAV); e, dessa forma, no presente trabalho, o nível de ruído foi mensurado utilizando-se ambos os recursos. Os dados foram avaliados por meio de análise estatística descritiva. A pesquisa teve aprovação na Comissão de Ética no Uso de Animais, $n^{0}$ 063, de 2016, e no Comitê de Ética em Pesquisa, ${ }^{\circ}$ 1907748, de 06/02/2017.

\section{RESULTADOS E DISCUSSÃO}

Foram avaliados 439 cães individualmente, de um total de 830, provenientes de 16 abrigos municipais. Houve mediana de 37,5 cães por abrigo, variando entre quatro e 197 cães. Entre o total de cães avaliados dos 16 abrigos, $17 \%$ (75/439) eram cães jovens entre seis meses e dois anos de idade, 62\% (272/439) eram cães adultos entre três e seis anos e 21\% (92/439) eram cães idosos acima de sete anos de idade; sendo que, em um abrigo, 100\% (36) dos cães eram idosos. Foram identificados quatro tipos de acomodações distintas: canis; canis com solário; área externa cercada, na qual os cães ficavam soltos; e casinha em área externa, com os cães acorrentados à casinha ou acorrentados a um "trilho" junto à casinha.

As acomodações "área externa cercada e casinhas com correntes e/ou 'trilhos' " foram incluídas no protocolo de avaliação devido à realidade encontrada nas visitas aos abrigos do Paraná. De um total de 243 recintos existentes nos 16 abrigos, foram avaliados 165, sendo 131 
canis (59 internos e 72 com solário) de 15 abrigos, 17 áreas externas de oito abrigos, sete casinhas com cães acorrentados (provenientes de um abrigo) e 10 casinhas com cães acorrentados em "trilho" (provenientes de três abrigos). Em relação ao número de cães por acomodação, 30\% (48/165) ficavam em canis individuais, $10 \%$ (17/165) em canis de duplas, 22\% (36/165) em canis coletivos de 3 a 5 cães, 18\% (30/165) em canis coletivos com mais de seis cães, $10 \%$ $(17 / 165)$ eram áreas externas cercadas com mais de seis cães soltos, 10\% (17/165) eram casinhas com cães acorrentados.

Para o princípio de boa alimentação, é necessário que haja ausência de fome e sede prolongadas. Para tanto, foram avaliados condição corporal, alimentação e suprimento de água. $\mathrm{Na}$ avaliação do escore de condição corporal (ECC) dos cães, $383(87 \%)$ cães estavam com escore adequado. Doze abrigos mantinham seus cães com ECC adequado. Os outros quatro abrigos mantinham mais de $23 \%$ dos seus cães com EEC inadequado; um mantinha um (25\%) cão obeso, um mantinha cinco (55\%) cães muito magros, porém em reabilitação, um mantinha sete $(23 \%)$ cães obesos e um abrigo tinha um (2\%) cão obeso e 19 (32\%) muito magros.

Todos os abrigos ofereciam ração seca e tinham ração para cães adultos. Quatro (25\%) abrigos tinham ração para filhotes e nenhum possuía ração para cães idosos, mesmo o abrigo que possuía $100 \%$ dos cães nessa faixa etária. Apenas um (6\%) abrigo possuía ração específica para cães em tratamento clínico. Sete (44\%) abrigos ofereciam ração aos cães uma vez ao dia, quatro $(25 \%)$ ofereciam duas vezes ao dia e cinco $(31 \%)$ abrigos ofereciam ração ad libitum. Foi observado um abrigo com 33\% (20/60) dos cães com escore corporal inadequado por caquexia.

Segundo o respondente, a ração era oferecida duas vezes ao dia, mas o tratador alegou que era possível fazer apenas uma vez ao dia e em quantidade de ração insuficiente para a quantidade de cães, caracterizando sofrimento animal por fome e desnutrição. Em outro abrigo, $23 \%$ (7/30) apresentaram escore corporal inadequado por obesidade. Neste abrigo, o respondente alegou desobediência dos tratadores em oferecerem a quantidade de alimento orientada. Controlar diariamente o número e o porte dos cães alojados permite calcular a quantidade de alimento que deve ser disponibilizado, o que garante fornecimento adequado e indica boa gestão quanto ao manejo nutricional.

A falta de alimento, além de ser um fator estressante, implica baixo grau de bem-estar animal, contribui para desencadear doenças (Capelli et al., 2016) e configura crime de maustratos. Capacitar os tratadores, supervisionar periodicamente o fornecimento de alimento e proporcionar exercício físico diário aos cães obesos reduz o desperdício de alimento e dinheiro público, além de garantir mais saúde e maior grau de bem-estar a esses cães. Além disso, os abrigos devem providenciar ração específica para todas as faixas etárias dos cães que mantêm.

Para a avaliação de ausência de sede prolongada, foram identificados $80 \%$ (132/165) dos recintos com o suprimento de água adequado, pois os bebedouros estavam funcionando, eram limpos e seguros e a água era limpa. A higienização diária dos bebedouros deve ser corrigida em três abrigos, cujos recintos totalizavam 39 bebedouros de uso coletivo sujos com limo ou fezes. Além de imprópria para o consumo, neste caso a água se torna um importante veículo de doenças. Meireles et al. (2008) mostraram que houve uma ocorrência de $15 \%$ a mais de Giardia duodenalis em cães de abrigos, comparados a cães domiciliados, sendo essa doença, assim como outras infecciosas, de transmissão oral.

Para o princípio de boa acomodação, é necessário que haja conforto na área de descanso, conforto térmico e facilidade de locomoção dos cães. Para tanto, foram avaliados a presença e a condição das camas, a presença de áreas pontiagudas nos recintos, a limpeza do pelame dos cães, o número de animais latindo incessantemente, tremendo por frio ou ofegante por conta do calor, além do espaço disponível nos recintos pela quantidade e pelo peso dos cães alojados.

Dos 165 recintos, $42 \%(69 / 165)$ tinham camas adequadas, $31 \%(52 / 165)$ tinham camas inadequadas, por estarem sujas, molhadas ou em quantidade inferior a uma por animal, e em $27 \%$ (44/165) dos recintos a cama era inexistente. Foram identificadas superfícies pontiagudas em 
$13 \%(22 / 165)$ dos recintos, provenientes de cinco abrigos, sendo esses recintos locais de risco para a segurança dos animais alojados, pois os cães podem se ferir nas instalações. Esse achado mostra que essas instalações estavam comprometidas, necessitando de manutenção e reparos. $\mathrm{Na}$ avaliação da condição da pelagem dos cães, 375 (86\%) apresentavam pelagem limpa e seca, livre de urina e fezes. Seis abrigos mantinham $100 \%$ dos seus cães com pelagem limpa, e oito abrigos, acima de $80 \%$. Wagner et al. (2014) mostraram a probabilidade de 72 e $77 \%$ dos cães de abrigos defecarem e urinarem, respectivamente, no lado oposto à cama, mostrando a forte preferência dos cães em expressarem o comportamento de eliminação em espaços distintos da alimentação e da área de descanso. Recintos pequenos ou lotados não permitem esse comportamento, comprometendo o bem-estar dos animais e a limpeza do pelame.

A limpeza da pelagem também pode ser um bom indicativo de limpeza ambiental e de camas adequadas (Barnard et al., 2015). A Associação de Veterinários de Abrigos (ASV) sugere que haja uma medida mínima de $60 \mathrm{~cm}$ entre essas três áreas distintas e enfatiza a importância para o bem-estar de qualquer animal dispor de um local macio para descanso e repouso, a fim de lhes proporcionar conforto e evitar lesões em pontos de apoio (Newbury et al., 2018). No presente estudo, foi possível observar, na maioria dos abrigos, falha da gestão em proporcionar aos cães um local confortável para descanso, comprometendo, assim, o grau de bem-estar dos cães para esse critério.

A maioria dos abrigos mantinham recintos limpos e cães com pelagem limpa; no entanto, nos abrigos que tinham seus cães com pelagem suja de urina ou fezes, a avaliação clínica e o tratamento dos animais devem ser realizados, assim como deve-se aumentar a frequência de limpeza dos recintos, bebedouros e comedouros, diminuir a população de cães mantidos nos recintos lotados, por meio de recolhimento seletivo e ações que incentivem a adoção dos animais, e disponibilizar camas adequadas. Quanto ao conforto térmico, a temperatura ambiente registrada variou de $17^{\circ} \mathrm{C}$ a $29^{\circ} \mathrm{C}$, com média de $22^{\circ} \mathrm{C}+4$; a umidade variou de $64,5 \mathrm{RH}$ a 104,2 RH, com uma média de 79+10.

A American Veterinary Medical Association (Companion..., 2008) recomenda que a temperatura ambiente para cães seja mantida entre $15,5^{\circ} \mathrm{C}$ e $26,6^{\circ}$ e a umidade relativa deve variar de 30 a $70 \%$. Os abrigos que apresentaram cães com desconforto térmico mantinham os cães em área externa cercada, canis com solário ou acorrentados em casinha. Havia 10 abrigos com cães apresentando desconforto térmico por calor, em dias acima de $25^{\circ} \mathrm{C}$, e dois abrigos com cães apresentando desconforto térmico por frio, em dias abaixo de $19^{\circ} \mathrm{C}$. Dos quatro cães que apresentaram desconforto térmico por frio, três eram de um mesmo abrigo, idosos e com lesões de pele, e a visita se deu em um dia de garoa.

Houve uma proporção de três cães <20kg para cada cão $>20 \mathrm{~kg}$ nos abrigos. Em relação ao espaço disponível para locomoção, do total de 165 recintos avaliados, $110(67 \%)$ tinham metragem adequada em relação à quantidade $\mathrm{e}$ ao peso dos cães alojados. Todas as áreas externas cercadas tinham metragens adequadas. Os cães amarrados em casinha, ou presos em "trilhos", têm baixo grau de bem-estar, pela condição restrita de se locomover e de expressar outros comportamentos sociais e da espécie, além de ficarem expostos às intempéries. $\mathrm{O}$ CRMV-PR (Guia..., 2016) não recomenda que cães sejam mantidos acorrentados em abrigos, assim como a AVS considera esse método de manutenção inaceitável para qualquer animal, em abrigos humanitários (Newbury et al., 2018).

Para o princípio de boa saúde, é necessário atender os critérios de ausência de ferimentos, de doenças e de dor. Para tanto, avaliou-se condição da pele e presença de claudicação, evidências de dor e de tosse nos cães e de diarreia nos recintos, bem como dados quanto à mortalidade e à morbidade dos cães no último ano. Na mensuração da condição da pele, 354 (81\%) cães não apresentavam nenhuma alteração. Houve uma média de $5+4(21 \%)$ cães por abrigo com alguma alteração, sendo sarna a causa mais provável. Houve um cão com provável tumor venéreo transmissível, caso não observado pelo profissional responsável pelo abrigo.

Foram observados $33(7 \%)$ cães apresentando sinal de desconforto e dor, provenientes de 11 (69\%) abrigos. As causas de sinais de dor foram diversas. Trinta e nove (9\%) cães apresentavam claudicação, sendo dois casos de grau severo, incluindo uma cadela gestante com fratura de membro torácico, sem atendimento veterinário. 
Por outro lado, em outro abrigo, um cão paraplégico era levado para passear duas vezes ao dia, em cadeira de rodas adaptada. $\mathrm{Na}$ avaliação para ausência de doenças, foi observado um $(0,2 \%)$ cão idoso com apatia, tosse e dispneia grave. O responsável relatou que o animal não estava em tratamento, mas que encaminharia para uma clínica veterinária conveniada. Quatro abrigos apresentaram 100\% (9/9), $67 \%$ (2/3), 38\% (3/8) e 29\% (4/12), respectivamente, dos recintos com diarreia no piso.

As alterações descritas podem indicar cuidados inadequados desses animais em situação de abrigo, problema de saúde ou má adaptação dos cães em situação de confinamento. A diarreia pode ser um indicador de desordem entérica ou estresse (Petersen et al., 2008), sendo importante o correto diagnóstico para a promoção das devidas adequações. A tosse não foi um sinal clínico representativo nos cães de abrigos do Paraná, podendo ter sido quase inexistente pela época do ano ou pelo fato de a maioria dos cães estarem abrigados em áreas bem ventiladas.

Em relação a cirurgias, quatro abrigos (25\%) realizavam procedimentos cirúrgicos, incluindo a esterilização, nos próprios estabelecimentos; oito (50\%) terceirizavam o serviço via convênio ou parcerias com universidades de veterinária, clínicas particulares, castramóvel ou com o auxílio de protetoras e organizações não governamentais; e quatro abrigos (25\%) não providenciavam a realização de cirurgias por não considerarem de sua responsabilidade. Para tratamento e separação dos cães doentes dos sadios, 37\% (6/16) dos abrigos tinham instalação que possibilitava essa divisão, mas apenas cinco o faziam; em 44\% (7/16) não havia recintos específicos, mas eram improvisados; e nos demais $19 \%$ (3/16), os animais em tratamento permaneciam sempre juntos com os sadios.

Em relação à utilização de protocolos para tratamento de dor, 19\% (3/16) dos abrigos alegaram não possuir, e $81 \%$ (13/16) abrigos alegaram possuir medicamentos para promover analgesia. Destes, 31\% (4/13) abrigos o faziam de forma adequada, possuíam medicamentos analgésicos no abrigo, e não foi identificado nenhum cão com dor. Os demais 69\% (9/13) foram classificados como inadequados, sendo $11 \%$ (1/9) pela inexistência de fármacos no estabelecimento, e $89 \%$ (8/9) pela falha na identificação de cães com dor, os quais foram diagnosticados pelo pesquisador e não estavam em tratamento. A falha de diagnóstico e de tratamento da dor indica baixo grau de bem-estar animal (Broom, 1991). A aquisição de medicamentos adequados e a capacitação para diagnóstico da dor devem partir dos esforços dos responsáveis pelo abrigo (Brasil, 2018). Manter animais em sofrimento por inobservância é infração ética, segundo o Código de Ética do Médico Veterinário, e configura crime de maustratos a animais (Res.1236/2018).

Os dados relacionados à população média e a média de mortalidade nos 12 últimos meses foram possíveis de serem obtidos em 31\% (5/16) dos abrigos, devido à presença de identificação dos animais e de dados cadastrais. Não foi possível obter, em nenhum abrigo, os registros de dados referentes a gastos com tratamento clínico dos cães. A falta de dados referentes aos gastos com morbidade e ao registro de mortalidade indica falha de identificação e de registro dos cães alojados, sendo o registro do prontuário uma obrigatoriedade dos médicos veterinários responsáveis, segundo o Código de Ética do Médico Veterinário (Res.1138/2016).

Os abrigos têm capacidade limitada para a prestação de cuidados aos animais, que dependem de fatores como número e estado dos animais admitidos, instalações e recursos, número e capacitação dos funcionários, tempo de estadia dos animais, entre outros (Newbury et al., 2018). Dessa forma, o controle do número de animais em um abrigo é fundamental para que seja possível o repasse de verba pública suficiente para garantir a manutenção adequada dos animais e da instalação.

Para o princípio do comportamento apropriado, deve-se atender aos critérios de expressão de comportamento social e de comportamentos naturais da espécie, relação humano-animal e o estado emocional. Para tanto, avaliou-se como os cães eram acomodados, sua rotina de exercícios, presença de comportamento anormal e latidos excessivos, sua reação à presença humana desconhecida e os comportamentos mais expressados pela maioria dos cães em cada abrigo. Em relação a exercícios, em 25\% (4/16) dos abrigos, todos os cães eram soltos em uma área cercada, diariamente; em $31 \%$ (5/16) dos 
abrigos, alguns cães eram soltos esporadicamente; em $37 \%$ (6/16) dos abrigos, os cães nunca eram soltos; e em 6\% (1/16) dos abrigos, todos os cães saíam para passear em guia diariamente. Em relação ao nível de latidos/barulho, dos 439 cães avaliados, 43 (10\%), provenientes de 14 abrigos, latiam incessantemente durante a visita.

Houve média de $27 \%$ dos recintos com cães latindo. A mensuração do nível de ruído nos abrigos pela EAV atingiu de 17 a $109 \mathrm{~mm}$, com média de $56 \mathrm{~mm}+25 \mathrm{~mm}$, sendo seis abrigos acima dessa média; a mensuração pelo equipamento Medidor Multifuncional THDLA 500 registrou nível de ruído de 27 a 115 dBA, com média de $66 \mathrm{dBA}+14 \mathrm{dBA}$, sendo oito abrigos acima dessa média. Os menores níveis de ruído em ambientes de abrigos sempre serão os mais desejáveis, visto que sua intensidade e duração comprometem severamente o bem-estar dos animais, podendo exceder $100 \mathrm{db}$, e, acima de $90 \mathrm{db}$, já podem causar danos auditivos irreversíveis em humanos (Newbery et al., 2018).

Em relação à reação dos cães diante de presença humana desconhecida (do pesquisador), 60 (14\%) cães apresentaram medo e 21 (5\%) cães apresentaram comportamento agressivo defensivo e ofensivo. Os demais 358 (81\%) cães se mostraram amigáveis ou indiferentes à presença do pesquisador. O estado emocional dos cães foi baseado em como esses se comportavam e interagiam no ambiente do abrigo, e indicava o estado positivo dos abrigos. $\mathrm{Na}$ mensuração da escala analógica visual (EAV), todos os abrigos apresentaram a maioria dos cães em estado de alerta. Em 10 (62\%) abrigos, foram verificados pelo menos quatro adjetivos de comportamento positivo nos cães, como "brincalhão", "feliz", "amigável", "relaxado" e "descontraído", sendo apenas $20 \%$ (2/10) desses abrigos com alto nível de ruído.

Desses, a maioria dos seus cães era mantida coletivamente em recintos externos cercados. Os demais 38\% (6/16) dos abrigos apresentaram pelo menos três adjetivos de comportamento negativo, como "nervoso", "inseguro", "ansioso" e "barulhento", sendo 66\% (4/6) desses abrigos com alto nível de ruído. Dos oito abrigos com alto nível de ruído, seis abrigos eram instalações fechadas, compostas por canis ou canis com solário; sendo $66 \%$ (4/6) abrigos que mantinham os cães coletivamente; e $34 \%$ (2/6) que mantinham os cães em recintos individuais. O princípio de comportamento apropriado é obtido pelos critérios de expressão de comportamento social, de expressão de outros comportamentos naturais da espécie, da relação humano-animal e do estado emocional do animal.

As situações de confinamento não são previsíveis para os cães e, assim, frequentemente causam medo e estresse em curto período de tempo. Além disso, possibilitam o surgimento de comportamentos anormais repetitivos e/ou compulsivos, pela incapacidade de se ajustar no ambiente em que está inserido (Garner, 2005). Quatro cães apresentaram comportamento anormal repetitivo (estereotipia), em quatro abrigos distintos; todos eram mantidos em canis com solário, apenas um era mantido em canil individual, dois canis com metragem e cama adequadas, e os demais com metragem e/ou cama inadequadas. Foram identificados 39 cães em isolamento social, sendo o bem-estar comprometido ainda mais nos recintos com metragens inadequadas.

Manter cães em duplas ou grupos possibilita a expressão do comportamento social. Como resposta a essa condição, Mertens e Unshelm (2015) constataram que manter cães em grupo diminui significativamente o ruído nos abrigos, melhora a relação homem-animal, reduz $\mathrm{o}$ comportamento anormal, reduz as brigas entre animais e aumenta a possibilidade de adoção. Enriquecimentos ambientais têm efeitos benéficos para a diminuição de ruído em ambiente de abrigos, diminuindo a excitação e a vocalização dos cães ansiosos e promovendo mais comportamentos de repouso e tranquilidade, o que indica relaxamento dos cães (Graham et al., 2004; Garvey et al., 2017). Os recintos individuais devem ser utilizados pelo tempo mínimo necessário e apenas em situações especiais, como quarentena, tratamento clínico (Barnard et al., 2014) ou para cães que não se adaptem em grupo.

Mais de $80 \%$ dos cães mantidos em abrigos públicos do Paraná tinham perfil favorável para a adoção, pois expressavam comportamentos desejáveis pelos adotantes, tais como comportamento lúdico, docilidade, previsibilidade, não barulhentos e boa relação 
com o ser humano e com outros cães. Os cães agressivos, medrosos e com alteração comportamental deveriam passar por uma avaliação clínica criteriosa, a fim de se diagnosticar e tratar possíveis causas desses comportamentos. Aos cães que não respondessem ao tratamento, que representassem risco à equipe de trabalhadores e aos que estivessem em sofrimento na situação de abrigo, a submissão à eutanásia poderia ser recomendada (Guia..., 2013). As falhas identificadas nos abrigos devem ser sanadas, a fim de que o alto grau de bem-estar seja a resposta individual de todos os cães. Os abrigos que tinham responsáveis menos comprometidos apresentaram pontos críticos na alimentação, na sanidade, na acomodação e no baixo grau de bem-estar dos animais.

\section{CONCLUSÃO}

A identificação e o registro dos animais nos abrigos municipais do Paraná são falhas. Isso compromete todo o gerenciamento de recursos públicos necessários para a manutenção adequada dos animais e das instalações. Os pontos críticos que interferem para o baixo grau de bem-estar dos cães foram relacionados à negligência no fornecimento de camas adequadas e no diagnóstico e controle da dor. Por outro lado, a condição sanitária e as emoções expressadas pela maior parte dos cães, na maioria dos abrigos municipais do Paraná, sugeriram que esses animais estivessem interagindo de forma positiva no ambiente em que estavam inseridos no dia da visita, sendo esses indicadores positivos de bem-estar animal e de comportamentos desejáveis para adoção.

\section{REFERÊNCIAS}

ACESSO e utilização dos serviços de saúde, acidentes e violências. Brasil, Grandes Regiões e Unidades da Federação. Rio de Janeiro: IBGE, 2013. cap. Presença de animais no domicílio. p.26-27.

ARRUDA, E.C.; NORONHA, J.; MOLENTO, R.C.M.. et al. Características relevantes das instalações e da gestão de abrigos públicos de animais no estado do Paraná, Brasil, para o bemestar a animal. Arq. Bras. Med. Vet. Zootec., v.71, p.232-242, 2019.
BARNARD, S.C.; VELARDE, A.; VILLA, P.D. Shelter quality - welfare assessment protocol for shelter dogs. Salignan: IRSEA, 2014. 50p.

BARNARD, S.; PEDERNERA, C.; CANDELORO, L. et al. Development of a new welfare assessment protocol for practical application in long-term dog shelters. Vet. Rec., v.178, p.1-8, 2015.

BRASIL. Lei 9.605, de 12 de fevereiro de 1998. Sanções penais e administrativas derivadas de condutas e atividades lesivas ao meio ambiente, e dá outras providências. Diário Oficial da União, Brasília, DF, 13 fev. 1998. Seção 1, p.29.

BRASIL. Ministério da Ciência Tecnologia, Inovações e Comunicações. Resolução Normativa, n.37. Diretriz da prática de eutanásia. Brasilia: CONCEA. 2018. 49p. (Anexo).

BRASIL. Portaria Ministério da Saúde 1.138, de 23 de maio de 2014. Define as ações e os serviços de saúde voltados para vigilância, prevenção e controle de zoonoses e de acidentes causados por animais peçonhentos e venenosos, de relevância para a saúde pública. Diário Oficial da União, Brasília, DF, 26 mai. 2014. Seção 1, p.83.

BROOM, D.M. Animal welfare: concepts and measurement. J. Anim. Sci., v.69, p.4167-4175, 1991.

CALHAU, L.B. Meio ambiente e tutela penal nos maus-tratos contra animais. Rev. Jus. Navigandi, p.1-24, 2007. Disponível em: $<$ http://sisnet.aduaneiras.com.br/lex/doutrinas/ar quivos/Meio\%20Ambiente.pdf $>$. Acessado em: 12 nov. 2016.

CAPPELI, S.; MANICA, E.; HASHIMOTO, J.H. Importância dos aditivos na alimentação de cães e gatos: revisão. PubVet. v.10, p.212-223, 2016.

COMPANION animal care guidelines. Schaumburg: AVMA, 2008 Available in: <https://www.avma.org/KB/Policies/Pages/Com panion-Animal-Care-Guidelines.aspx $>$.

Accessed in: 12 Nov. 2018.

GARNER, J.P. Stereotypies and other abnormal repetitive behaviors: potential impact on validity, reliability, and replicability of scientific outcomes. ILAR J., v.46, p.106-117. 2005. 
GARVEY, M.; STELLA, J.; CRONEY, C. Auditory stress: implications for kenneled dog welfare. Center of animal welfare science. Department of Comparative Pathobiology, College of Veterinary Medicine, Purdue University. Expert reviewed. 2017. Available in: $<$ https://extension.purdue.edu/extmedia/VA/VA18-W.pdf>. Accessed in: 7 Mar. 2018.

GRAHAM, L.; WELLS, D.L.; HEPPER, P.G. The influence of olfactory stimulation on the behaviour of dogs housed in a rescue shelter. Lab. Dermaph., 2004. Available in: <http://www.dermapharm.com.pl/pl/a/literaturaolfactory-stimulation-influence $>$. Accessed in: 14 Mar. 2018.

GUIA brasileiro de boas práticas para eutanásia em animais - conceitos e procedimentos recomendados. considerações gerais: conceito de eutanásia e suas indicações. Brasília: CFMV, 2013. cap.1, p.14-15.

GUIA de controle humanitário da população canina. São Paulo: ICAM, 2007, p.24.

GUIA técnico para construção e manutenção de abrigos e canis. Orientação técnica referentes à estrutura física. Curitiba: CRMVPR, 2016. p.1014.
HAMMERSCHMIDT, J.; MOLENTO, C.F.M. Análise retrospectiva de denúncias de maustratos contra animais na região de Curitiba, Estado do Paraná, utilizando critérios de bemestar animal. Braz. J. Vet. Res. Anim. Sci., v.49, p.431-441, 2012.

MEIRELES, P.; FERREIRA, F.M.; SOCCOL, V.T. Survey of giardiosis in household and shelter dogs from metropolitan areas of Curitiba, Paraná state, Southern Brazil. Vet. Parasitol., v.152, p.242-248, 2008.

MERTENS, P.; UNSHELM, J. Effects of group and individual housing on the behavior of kennelled dogs in animal shelters. J. Anthrozoös, v.9, p.40-51, 2015.

NEWBURY, S.; BLINN, M.K.; BUSH, P.A. et al. Diretrizes sobre os padrões de cuidados em abrigos de animais. São Paulo: Associação de veterinários de abrigos, 2018.94p.

PETERSEN, C.A.; DVORAK, G.; SPICKLER, A.R. Principles of infection control for animals. cap2. In: _. Shelters infection control manuela for animal shelters for veterinary personnel. Iowa: Maddie, 2008. 136p.

WAGNER, D.; NEWBURY, S.; KASS, P.; HURLEY, K. Elimination behavior oh shelters dogs housed in double compartment kennels. Plos Med. J., v.9, p.1-9, 2014. 